\title{
BMJ Open MyBackPain-evaluation of an innovative consumer-focused website for low back pain: study protocol for a randomised controlled trial
}

\author{
Leanne M Hall, ${ }^{1}$ Manuela Ferreira, ${ }^{2}$ Jenny Setchell, ${ }^{1}$ Simon French, ${ }^{3}$ \\ Jessica Kasza, ${ }^{4}$ Kim L Bennell, ${ }^{5}$ David Hunter, ${ }^{\circ}$ Bill Vicenzino, ${ }^{7}$ Chris Dickson, ${ }^{8}$ \\ Paul Hodges ${ }^{1}$
}

To cite: Hall LM, Ferreira M, Setchell J, et al. MyBackPainevaluation of an innovative consumer-focused website for low back pain: study protocol for a randomised controlled trial. BMJ Open 2019;9:e027516. doi:10.1136/ bmjopen-2018-027516

- Prepublication history for this paper is available online. To view these files, please visit the journal online (http://dx.doi org/10.1136/bmjopen-2018027516).

Received 26 0ctober 2018 Revised 8 February 2019 Accepted 11 April 2019
Check for updates

(C) Author(s) (or their employer(s)) 2019. Re-use permitted under CC BY-NC. No commercial re-use. See rights and permissions. Published by BMJ.

For numbered affiliations see end of article.

Correspondence to

Prof. Paul Hodges;

p.hodges@uq.edu.au

\section{ABSTRACT}

Introduction Despite the prevalence of low back pain (LBP) worldwide, many people with the condition do not receive evidence-based care or achieve the best possible outcomes. There is a gap in the dissemination of evidence-based information across the globe. The advent of the internet has changed the way people obtain health information. As such, trustworthy, tailored and validated LBP resources may help bridge the gap. This study aims to measure the effectiveness of a new website (MyBackPain) in improving spinal health literacy, treatment preferences and clinical outcomes for people with LBP, in comparison with other online resources.

Methods and analysis This online, pragmatic, randomised controlled trial will comprise 440 people with non-specific LBP of any duration. In addition to access to publicly available online information (control group), the intervention group will be given access to the MyBackPain. org.au website. Participants and research staff, including the biostatistician, will be blinded to treatment allocation. Data will be collected at baseline, 1, 3 (primary end-point), 6 and 12 months via online surveys and questionnaires. The primary outcome is spinal health literacy. Secondary outcomes include quality of treatment preferences (stated and observed) and LBP clinical outcomes (pain, disability and quality of life). Analyses will be by intention-to-treat and include outcome data on all randomised participants. Descriptive statistics will be presented for demographic and clinical characteristics.

Ethics and dissemination This trial has been prospectively registered with the Australian New Zealand Clinical Trials Registry and has ethical approval from the University of Queensland Human Research Ethics Committee (2017000995). Trial outcomes will be shared via national and international conference presentations and peer-reviewed journal publications.

Trial registration number ACTRN12617001292369; Preresults.

\section{INTRODUCTION}

According to the global burden of disease study, low back pain (LBP) is the leading cause of disability worldwide ${ }^{1}$ placing an enormous
Strengths and limitations of this study

- Involves the collaboration of individuals with low back pain, clinicians and international experts in back pain to conceptualise a new evidence-based low back pain website.

- An entirely online randomised clinical trial that allows engagement of people with low back pain across Australia to increase generalisability of the results.

- Contamination of study groups is not high risk given the password-protected website and specific instruction to participants not to share site information but we cannot measure adherence to this request.

burden on individuals and economies. Up to $80 \%$ of individuals experience LBP at least once in their lifetime ${ }^{2}$ and when it persists it accounts for $30 \%$ of all chronic pain. ${ }^{3}$ Total annual expenditure in Australia includes approximately $\$ 4.8$ billion in direct costs ${ }^{4}$ and over $\$ 8$ billion in indirect costs. ${ }^{5}$ Much of this burden involves unnecessary and ineffective assessments and treatments. ${ }^{6}$ The impact of LBP is worsened by negative messages and beliefs, and poor-quality management. ${ }^{7} 8$ Early education and access to the most effective treatments could reduce much of this excessive burden. There is a clear role for a LBP education/guidance portal to empower patients to optimise active participation in their negotiation of treatments and healthcare providers.

People increasingly use the internet to obtain health-related information. ${ }^{9}{ }^{10}$ It has great potential to educate and engage patients in the management of health conditions. People with LBP consistently report a desire for trustworthy information about their condition ${ }^{11-14}$ and the internet, with its capacity to provide tailored information in 
varied formats at a time and place of the user's choosing, is ideal to provide such information. Positive features of internet use include the potential for patients to become better informed about their condition and potential treatment options, ${ }^{15-18}$ to become more engaged with their treatment ${ }^{19}$ and to improve health outcomes with more appropriate use of health resources. ${ }^{1820} 21$ Access and reflection on information prior to clinical visits could also ensure efficient use of clinical consultation time, ${ }^{1520}$ enhance relationships between patients and clinicians ${ }^{18}$ and foster informed decision-making. ${ }^{20}$

When evaluated against criteria developed from relevant guidelines and research evidence, ${ }^{22-24}$ LBP websites are consistently rated as 'poor' in overall quality and do not meet the expressed needs of patients with LBP (Nielsen, 2017 unpublished data). In addition, the language and terminology used on many LBP websites are not tailored to the intended audience making the information difficult for users to understand. ${ }^{23}$

We have shown that people with LBP are interested in a range of information topics including diagnostic and treatment information, lay or experience-based information, practical self-help strategies, recognition and discussion of psychosocial concerns. ${ }^{25}$ These are often lacking in current websites (Nielsen, 2017 unpublished data). Consumer preferences regarding presentation of information emphasise multimodality, readability, quality assurance and interactivity (Nielsen, 2017 unpublished data), none of which are satisfactorily achieved with the resources currently available.

As a part of this randomised controlled trial, we have developed a comprehensive LBP website (MyBackPain. org.au) that integrates evidence-based LBP information and tailored guidance and explicitly considers the needs and preferences of individuals with LBP. The highest quality information for people with both acute and chronic LBP has been identified and distilled to easily understood resources in multiple formats (patient and clinician videos, information sheets, quizzes) and uses evidencebased algorithms to create tailored consumer guidance. The MyBackPain website is designed to improve health outcomes by: (1) enhancing consumer confidence in managing their condition and making treatment choices with emphasis on evidence-based assessments and treatments, and avoidance of investigations and treatments that are ineffective, unnecessary or harmful; (2) de-medicalising and normalising LBP with messages in multiple formats that reinforce that back pain is a natural part of life for many and in most cases can be managed with early return to activity; (3) providing tools for individuals to identify if further investigation and/or management may be required and (4) engaging patients in healthy behaviours and attitudes to reduce the burden of LBP.

\section{Aim}

The aim of this randomised controlled trial is to evaluate the effectiveness of the newly developed, multifaceted MyBackPain website compared with existing internet resources. We hypothesise that the MyBackPain website will be more effective than existing internet resources in improving health literacy, choice of evidence-based treatments and clinical outcomes in people with LBP.

\section{METHODS AND ANALYSIS \\ Study design}

This manuscript describes a research protocol for the MyBackPain randomised controlled trial. This prospectively registered, pragmatic, online-based, randomised controlled trial with assessor and participant blinding, will recruit individuals with LBP from across Australia. Participants will be randomised to groups that could either; (1) access any existing online resources (control group) or (2) have access to the MyBackPain online resource in addition to other readily available online resources (intervention group). The comparison with self-directed use of the internet will provide a pragmatic comparison of the effects of the MyBackPain website. Central, computerised randomisation will be used to ensure allocation concealment. This protocol has been developed in accordance with Standard Protocol Items: Recommendations for Interventional Trials. ${ }^{26}$

\section{Participant recruitment}

Participants will be recruited from the community using newsletters, email lists, consumer groups (eg, Arthritis Australia, Chronic Pain Australia), websites, social media and talks at group meetings. Our partner health insurer (Medibank Private) will also make available their insurance membership cohort for recruitment purposes. This study will recruit participants with LBP from November 2017 until the sample size is achieved.

Potential participants will be provided with a web-link to a page that provides the participant information sheet and consent form where they will be asked if they have read and understood the information and if they consent to participate in the trial. Consent will be provided by checking the appropriate box. Those who consent to participate will then be directed to a screening form to determine their eligibility. Eligible participants will complete the baseline data questionnaires/surveys before randomisation into the intervention or control group. To encourage retention in the study, participants will be offered entry into a draw for an iPad mini on completion of all time points of the data collection. One iPad will be awarded for each 44 participants.

\section{Participants}

\section{Inclusion criteria}

Participants will be included if they meet all the following criteria:

- Current LBP of any duration.

- Aged 18 years and above (no upper age limit).

- Reside in Australia.

- Adequate English to complete outcome measures and interact with the MyBackPain website. 
- Internet access for the duration of the trial.

Participants will be excluded if they have a previous or existing serious spinal pathology (defined as fracture, cancer, infection) or been diagnosed with specific spinal pathology including sciatica, lumbar spinal stenosis or nerve root compromise.

\section{Study treatments}

Participants will be randomised to an intervention or control group. Stratified permuted block randomisation will be used, with blocks of sizes $6-12$ stratified by symptom duration (acute or chronic). An episode of acute LBP will be considered to be pain of less than 6 weeks' duration with at least 4 weeks between pain episodes. ${ }^{27}$ All other pain presentations will be considered chronic LBP. All participants will be advised that the study aims to investigate the impact of the use of the internet on LBP. Participants are free to use web resources in any manner in which they feel appropriate and for any amount of time.

\section{Intervention: MyBackPain website}

The content and framework of the MyBackPain website has been developed according to an extensive process of consultation and collaboration with individuals with LBP and clinicians, and with an international team of experts who were engaged to contribute to the development of evidence-based content (table 1).

Table 1 Steps involved in the development of the MyBackPain website

\begin{tabular}{ll} 
Step & Process \\
\hline 1. Identification of consumer needs - website & Qualitative study of consumer needs involving focus groups and interviews with patients
\end{tabular}
content and presentation with $\mathrm{LBP}^{25}$ and clinicians ${ }^{32}$

2. Evaluation of existing LBP websites

Review comparing content of existing websites to content and format criteria developed from step 1 (Nielsen et al, unpublished data).

3. Establishment of expert steering committee

An international advisory committee established with representation of multiple disciplines (medicine; physiotherapy; chiropractic; occupational therapy, etc) and multiple regions (Australia; Europe; North America; Asia).

4. Identification of key messages

Evidence-based messages were identified from the literature (clinical practice guidelines: systematic reviews). Experts were consulted using a Delphi process to review, add, edit and refine the key messages. Language was optimised with consumer focus groups. Priority order of presentation was assessed using an on-line process with consumers and international experts from multiple disciplines. A final list of 30 messages was identified for reinforcement throughout all materials on the website and all formats (French et al, 2017 unpublished data).

5. Generation of list of frequently asked questions

6. Content consensus

7. Development of treatment summaries

8. Profession descriptions

9. Content development content utilisation

11. Consumer input, review and feedback

12. Beta testing

\section{Qualitative study with focus groups.}

Consensus workshop at 'LBP Forum' international conference.

Orthodox and complimentary treatments were identified by the expert steering committee with consumer input. A draft description of each treatment and a synthesis of research evidence from the best available evidence (systematic reviews; clinical trials and clinical practice guidelines) was developed by an independent expert group and a consumer writer. International experts were identified to review each treatment summary and allocate an 'evidence grade badge' to enable quick identification of evidence levels for treatments or the potential for harm. All summaries and evidence grades were reviewed for consistency by the international advisory board and six additional experts.

Consultation with respective professional societies.

Content was developed in a range of formats including: an algorithm aligned to that used by clinicians to tailor information for people with acute and chronic LBP and create a management plan; multilayered information content enabling users to access as little or as much detail on a topic as they prefer; self-monitoring applications to track status and recovery as determined by measures of activity and participation; and responses to the 'frequently asked questions'. All content was aligned to the 'key messages'.

Two algorithms were developed on the basis of existing stratification/prognostic tools. The StartBack tool and Pick-up tool were adapted to guide the user experience for individuals with LBP of greater than or less than 3 months duration, respectively. The tools were used to evaluate possible risk of poorer outcome and tailoring information regarding advice to access psychologically informed resources if required.

Consumers contributed to focus groups in the planning phases and review and refinement of content in the latter phases. Professional groups with an interest in LBP and relevant consumer groups were consulted to assist with refinement of the website content. Extensive testing of formats and information was undertaken using a variety of methods including focus groups.

A full beta version of the website was constructed and extensively reviewed with consumer feedback.

LBP, low back pain. 
Participants randomised to the intervention group will be given access to the MyBackPain website for the duration of the trial via a unique username and password to minimise crossover from the control group to the intervention group. The website is not publicly available and no content can be accessed without the username/password combination individually provided to participants in the intervention group, with a request not to share the website or its content with others. Participants will be able to use the website in multiple ways: self-directed browsing and searching of the content; inbuilt automated guided content tailored to the features of their presentation and identified information priorities; and the opportunity to 'opt-in' to receive regular emails that highlight key messages about LBP. Participants will be free to determine how, when and how often they access the website. They will be free to decide which content they use and the format they prefer (eg, text, video, patient stories etc). The website will send automated messages to encourage users to return and access additional content and refresh their knowledge if they have opted to receive the regular emails. They will be encouraged to save information of interest to their 'dashboard' for easy access and print out relevant information for later reference or use in visits with their healthcare provider.

\section{Control}

Participants randomised to the control group will be asked to record the address of any websites they access for information about LBP throughout the trial and relay this information in the weekly (weeks 1-12) and monthly (months 3-12) online diaries. They will not have access to, or knowledge of, the MyBackPain website until it is launched to the public (ie, after completion of the trial).

\section{Data collection}

All data will be collected online using REDCap (Research Electronic Data Capture, Vanderbilt University). Online data collection was chosen to allow inclusion of participants from any location in Australia. This approach enhances the feasibility of the trial and the generalisability of the results. Participants will complete an online questionnaire at baseline to provide demographic data (eg, age, sex, height, weight, education, job and job status) and details about their low back symptoms (including location, intensity, duration, frequency and past treatments).

All other data will be collected at baseline, 1 month, 3 months (primary end-point), 6 months and 12 months (table 2). In addition, weekly diaries will be used to gather information about current pain levels, treatments used and websites visited for information about LBP. At the 3-month time point, the diaries will be sent monthly for the remainder of the trial. The time of primary outcome (3 months) has been selected as we expect access to the

\begin{tabular}{|c|c|c|c|c|c|c|c|}
\hline \multirow[b]{2}{*}{ Time point } & \multirow[t]{2}{*}{ Enrolment } & \multirow[t]{2}{*}{ Baseline } & \multirow{2}{*}{$\frac{\text { Allocation }}{0}$} & \multicolumn{4}{|c|}{ Postallocation (months) } \\
\hline & & & & 1 & 3 & 6 & 12 \\
\hline \multicolumn{8}{|l|}{ Enrolment } \\
\hline Eligibility screen & $\mathrm{x}$ & & & & & & \\
\hline Informed consent & $x$ & & & & & & \\
\hline Randomisation & & & $x$ & & & & \\
\hline \multicolumn{8}{|l|}{ Intervention } \\
\hline \multicolumn{8}{|l|}{ Control } \\
\hline MyBackPain & & & $\leftarrow$ & & & & $\longrightarrow$ \\
\hline \multicolumn{8}{|l|}{ Assessment } \\
\hline Demographics & & $x$ & & & & & \\
\hline HLQ & & $x$ & & $x$ & $x$ & $x$ & $x$ \\
\hline $\mathrm{RMDQ}$ & & $x$ & & $x$ & $x$ & $x$ & $x$ \\
\hline AQoL-8D & & $x$ & & $x$ & $x$ & $x$ & $x$ \\
\hline Treatment choices & & $x$ & & $x$ & $x$ & $x$ & $x$ \\
\hline \multicolumn{3}{|l|}{$\begin{array}{l}\text { Weekly diary-pain VAS, } \\
\text { websites visited, treatments } \\
\text { used }\end{array}$} & 4 & & 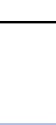 & & \\
\hline $\begin{array}{l}\text { Monthly diary-pain VAS, } \\
\text { websites visited, treatments } \\
\text { used }\end{array}$ & & & & & & . & \\
\hline
\end{tabular}

AQoL-8D, Assessment of Quality of Life - 8 Dimension; HLQ, Health Literacy Questionnaire; RMDQ, Roland Morris Disability Questionnaire; VAS, Visual Analogue Scale. 
resource to modify treatment choices and outcome over an extended period.

Data pertaining to the information sought and frequency of use of the intervention website will be collected using OpenTracker software and assessed via website-use statistics.

\section{Treatment adherence}

Each user's history of access to the MyBackPain website will be recorded based on their unique log-in (intervention group only), and the use of other websites will be recorded via participant entries into the online weekly diary.

\section{Patient and public involvement}

Patients with LBP were extensively involved in the development of the MyBackPain website. This involved a multistep process that included identification of consumer needs and preferences for content and presentation. Extensive testing of the website was undertaken with consumers. Patients were consulted for design of outcome measures of treatment preferences. Patients or public were not otherwise involved in study design. Participants will contribute to dissemination of the randomised controlled trial proposed in this protocol.

\section{Blinding}

Participants and investigators (except the project manager) will be blinded to treatment allocation. All participants will be advised that the study aims to investigate the impact of use of the internet on LBP, but will be unaware of which specific website will be evaluated. Data analyses will be conducted by a blinded biostatistician. We anticipate no reason for revealing a participant's intervention allocation during the trial.

\section{Outcome measures}

\section{Primary outcomes}

The primary outcome will be the spinal health literacy evaluation measured with the Health Literacy Questionnaire (HLQ) ${ }^{28}$ The HLQ includes 44 items and nine dimensions. Dimensions 2 and 3 will be included as co-primary outcome measures: 'having sufficient information to manage my health' and 'actively managing my health'. These dimensions will be assessed using a 4-point Likert scale (1-completely disagree; 4 -completely agree) and a $0-100$ score will be presented for each dimension. The preamble to the survey will ask participants to consider their LBP when answering the survey.

\section{Secondary outcomes}

Secondary outcomes include dimensions 1 ("feeling understood and supported by healthcare providers'), and 4-9 ('social support for health'; 'appraisal of health information'; 'ability to actively engage with healthcare providers'; 'navigating the healthcare system'; 'ability to find good health information' and 'understand health information well enough to know what to do', respectively) of the HLQ. These seven dimensions will be assessed using a 4-point Likert scale (1-completely disagree; 4-completely agree).

We will also investigate patient preference for a number of treatment choices mentioned in the MyBackPain website in terms of the likelihood of healthy (ie, treatments rated as strong evidence and some evidence) or unhealthy treatment choices. The treatments patients indicate as being healthy or unhealthy (stated) and the treatments they actually use (observed) will be measured in each group in three ways:

1. Quality of treatment preference (stated): Patient decision-making will be measured by evaluation of stated effectiveness of treatment choices. Patients will be asked to click on a 5-item scale (effective, somewhat effective, unsure, not very effective, not effective) if they think a subset of treatments discussed in the MyBackPain website are effective for people's LBP in general (but not specifically their own pain). Treatment choices will be scored against the recommendations provided in the MyBackPain website according to the classifications of 'good evidence', 'may work', 'not enough evidence', 'unlikely to work' and 'may be harmful'.

2. Quality of treatment preference (observed-scored): Treatments that are used by participants will be evaluated against the recommendations provided in the MyBackPain website according to the classifications of 'good evidence', 'may work', 'not enough evidence', 'unlikely to work' and 'may be harmful'. Participants of both groups will be asked to record weekly in the online diary any treatments received for their LBP.

3. Quality of treatment preference (observed-proportion): The proportion of participants who choose treatments that are, according to the MyBackPain website, either recommended or considered to have no effect or be harmful in each group will be assessed separately (recommended; no effect; harmful), using data from the online participant diary.

LBP clinical outcomes will also be included as secondary outcomes and measured with the following validated tools:

1. Pain-Visual Analogue Scale of average overall LBP in the last week recorded on a scale anchored with 'no pain' at 0 and 'worst pain imaginable' at 10.

2. Disability—Roland Morris Disability questionnaire. ${ }^{29}$

3. Quality of life-AQoL-8D. ${ }^{30}$

\section{Data integrity}

All data will be directly collected into a custom-built electronic data capture program, with a prompt for doublechecking of the accuracy of the primary outcomes. Any inconsistencies in the data will be explored and resolved. The database will be backed-up regularly on a secure network and be compliant with the International Council for Harmonisation (ICH) Guideline for Good Clinical Practice, ${ }^{31}$ according to our data management plan. Study personnel will only be able to access the database with a personal login and password. 
Table 3 Trial registration data

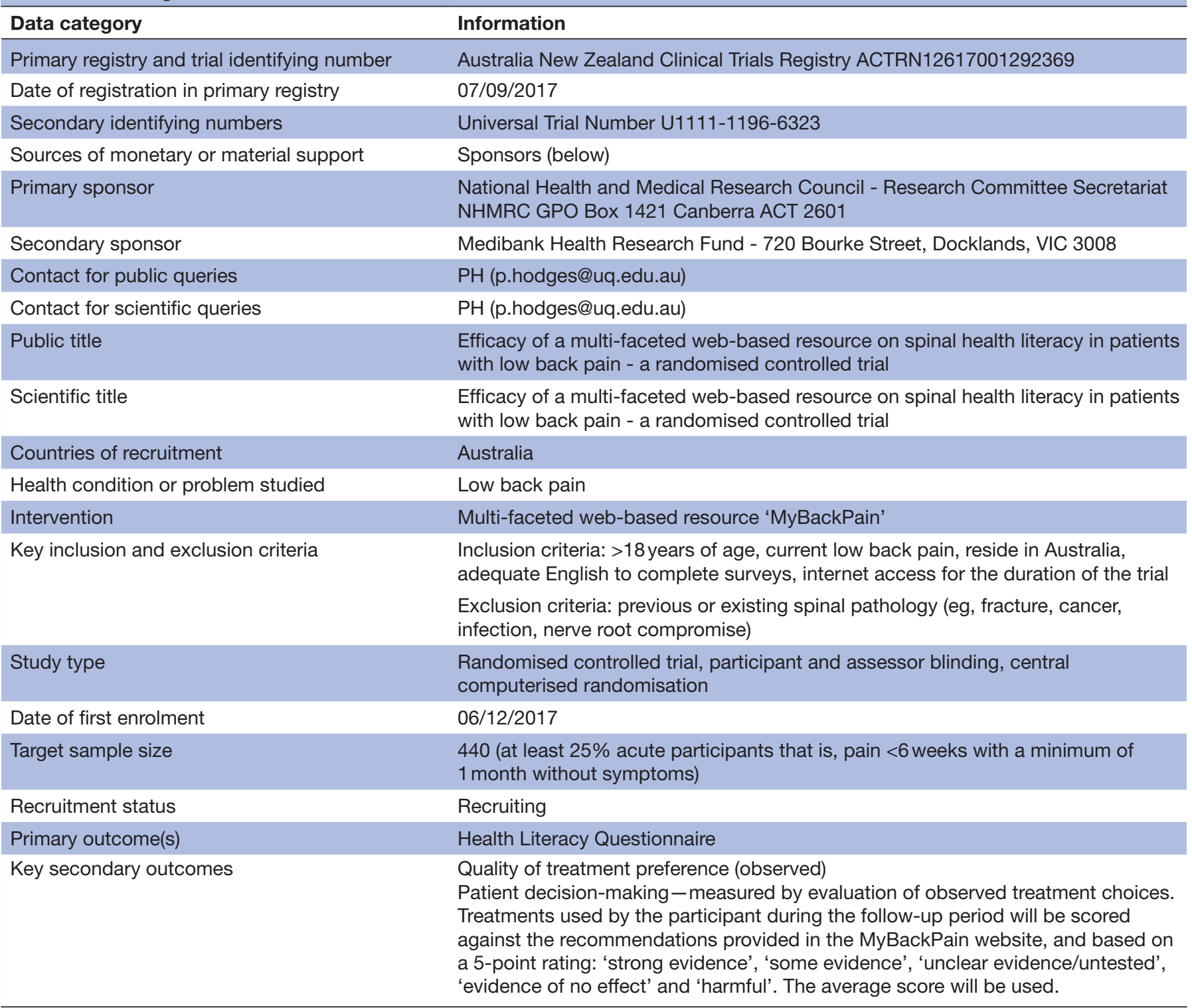

\section{Retention of documents}

The study investigators will maintain adequate and accurate records to enable the conduct of the study to be fully documented and the study data to be subsequently verified. Should the study investigators wish to assign the study records to another party or move them to another location, the sponsor will be notified in advance. After the completion of the study, study data will be archived by The University of Queensland for a minimum of 15 years.

\section{Data analysis}

Analyses will be by intention-to-treat of all randomised participants who completed the baseline surveys. To assess the difference in the primary outcome between groups, longitudinal linear regression models will be fit, including all data from 1, 3, 6 and 12 months as an outcome for each participant. Models will be adjusted for baseline values of outcomes and the stratification variable symptom duration and also include a term for month, and an interaction between month and randomised group included as fixed effects, with random effects for participants. Similar longitudinal logistic regression models will be used for binary outcomes. These models will be interrogated to yield differences between groups at each time point. Standard diagnostic plots will be used to assess regression assumptions. Descriptive statistics on demographics and clinical characteristics will be presented for both the control and intervention group as the mean change (SD, 95\% CI) or counts and percentages for categorical variables. Multiple imputation methodology will be employed to account for missing data. No statistical adjustment will be made for multiple testing. All tests will be carried out at the $5 \%$ level of significance.

Sample size calculation is based on an effect size of 0.30 , for 'having sufficient information to manage my health' and 'actively managing my health' dimensions of the HLQ. A sample size of 440 participants (minimum of 
$25 \%$ acute participants) will achieve $80 \%$ power to detect the desired effect size, allowing for a conservative loss to follow-up rate of $20 \%$ at 3 months.

\section{Ethics and dissemination}

The study is registered with the Australian New Zealand Clinical Trials Registry (table 3). The study is sponsored by the National Health and Medical Research Council of Australia and Medibank Health Research Fund and centrally managed by staff at the University of Queensland. The trial sponsor has had no role in the design or conduct of the trial. The current protocol is version 1 (7 September 2017), and any modifications to the protocol will require formal amendment following the approval of the principal investigator $(\mathrm{PH})$.

Participants will be provided with the contact details of the project manager for any queries or concerns. Any complaints arising from the trial will be recorded and acted on in accordance with institutional policy. Participants will be informed they are free to withdraw from the study at any time without consequence. They will be asked if they would like to receive a copy of the manuscript at the completion of the trial.

All data will be stored in electronic format in a de-identified manner on a secure server. The database will be password protected and only accessible by the research team. At the completion of the trial, the data collection portal will be closed and data will be retained in a de-identified format on the protected server at The University of Queensland. The MyBackPain website will remain active and launched to the public at the completion of the trial. Users of the MyBackPain website will have the option to create a user account on the website. This information will not be collected or used by the project team and will be housed on a host server managed by Arthritis Australia. Users of the MyBackPain website will be told of the purpose and protection of the user account prior to its creation.

We do not anticipate further use of the data, but participants will be asked to give consent to the potential future use of de-identified data so as not to limit this possibility. Any potential plan to use the data for an additional purpose will be considered by the investigative team.

Manuscript(s) will be submitted to major peer-reviewed journal(s) after the completion of randomised clinical trial. Leading multidisciplinary conferences on pain, LBP and primary care will also be targeted for dissemination of the study findings.

\section{Author affiliations}

${ }^{1}$ School of Health and Rehabilitation Sciences, The University of Queensland, Brisbane, Queensland, Australia

${ }^{2}$ Institute of Bone and Joint Research, University of Sydney, Saint Leonards, New South Wales, Australia

${ }^{3}$ Department of Chiropractic, Macquarie University, Sydney, New South Wales, Australia

${ }^{4}$ Department of Epidemiology and Preventive Medicine, Monash University, Melbourne, Victoria, Australia

${ }^{5}$ CHESM, University of Melbourne, Parkville, Victoria, Australia

${ }^{6}$ The University of Sydney, Sydney, New South Wales, Australia

${ }^{7}$ Physiotherapy, The University of Queensland, Queensland, Queensland, Australia
${ }^{8}$ Arthritis Australia, Sydney, New South Wales, Australia

Contributors LH contributed to design of the study; prepared the trial registration and application for ethical approval; drafted and revised the manuscript. MF designed and conceptualised the study; prepared the trial registration and application for ethical approval; drafted and revised the manuscript. JS designed and developed the MyBackPain website. SF contributed to designed and conceptualised the study; drafted and revised the manuscript. JK designed the data/statistical analysis plan. KB, DH, BV contributed to designed and conceptualised the study; revised the manuscript. CD designed and developed of the MyBackPain website. PH designed and conceptualised the study; designed and developed the MyBackPain website; prepared the trial registration and application for ethical approval; drafted and revised the manuscript. All authors reviewed and approved the final manuscript.

Funding This work was supported by the NHMRC (APP1079078) and a grant from the Medibank Health Research Fund (table 3). MF is funded by an NHMRC Career Development Fellowship (APP1143593) and a Sydney Medical Foundation Fellowship. DJH is supported by an NHMRC Practitioner Fellowship. KLB is supported by a NHMRC Principal Research Fellowship (APP1058440). JS is supported by an NHMRC Early Career Fellowship (APP1157179).

Competing interests DJH is on advisory boards for Merck Serono, Tissuegene, TLC Bio and Flexion, outside of the submitted work.

Patient consent for publication Not required.

Ethics approval University of Queensland Human Research Ethics Committee.

Provenance and peer review Not commissioned; externally peer reviewed.

Open access This is an open access article distributed in accordance with the Creative Commons Attribution Non Commercial (CC BY-NC 4.0) license, which permits others to distribute, remix, adapt, build upon this work non-commercially, and license their derivative works on different terms, provided the original work is properly cited, appropriate credit is given, any changes made indicated, and the use is non-commercial. See: http://creativecommons.org/licenses/by-nc/4.0/.

\section{REFERENCES}

1. Hoy D, March L, Brooks $P$, et al. The global burden of low back pain: estimates from the Global Burden of Disease 2010 study. Ann Rheum Dis 2014;73:968-74.

2. AloHa W. Australia's Health 2006. Canberra: AlHW, 2006.

3. Henderson JV, Harrison CM, Britt HC, et al. Prevalence, causes, severity, impact, and management of chronic pain in Australian general practice patients. Pain Med 2013;14:1346-61.

4. A problem worth solving [press release]. Elsternwick 2013.

5. Walker BF, Muller R, Grant WD. Low back pain in Australian adults: the economic burden. Asia Pac J Public Health 2003;15:79-87.

6. Buchbinder R, van Tulder M, Öberg B, et al. Low back pain: a call for action. Lancet 2018;391:2384-8.

7. Alyousef B, Cicuttini FM, Davis SR, et al. Negative beliefs about back pain are associated with persistent, high levels of low back disability in community-based women. Menopause 2018;25:977-84.

8. Carey M, Turon H, Goergen S, et al. Patients' experiences of the management of lower back pain in general practice: use of diagnostic imaging, medication and provision of self-management advice. Aust J Prim Health 2015;21:342-6.

9. Fox SJ. The Social Life of Health Information. Washington DC, 2009.

10. Kummervold PE, Chronaki CE, Lausen B, et al. eHealth trends in Europe 2005-2007: a population-based survey. J Med Internet Res 2008;10:e42.

11. Brown CA. The beliefs of people with chronic pain in relation to 'important' treatment components. Eur J Pain 2004;8:325-33.

12. Dewar A, White M, Posade ST, et al. Using nominal group technique to assess chronic pain, patients' perceived challenges and needs in a community health region. Health Expect 2003;6:44-52.

13. Glenton $\mathrm{C}$. Developing patient-centred information for back pain sufferers. Health Expect 2002;5:319-29.

14. Laerum E, Indahl A, Skouen JS. What is "the good backconsultation"? A combined qualitative and quantitative study of chronic low back pain patients' interaction with and perceptions of consultations with specialists. J Rehabil Med 2006;38:255-62.

15. Ahmad F, Hudak PL, Bercovitz K, et al. Are physicians ready for patients with Internet-based health information? J Med Internet Res 2006;8:e22.

16. Eysenbach G, Diepgen TL. Towards quality management of medical information on the internet: evaluation, labelling, and filtering of information. BMJ 1998;317:1496-502. 
17. Mclntosh A, Shaw CF. Barriers to patient information provision in primary care: patients' and general practitioners' experiences and expectations of information for low back pain. Health Expect 2003:6:19-29.

18. Wald HS, Dube CE, Anthony DC. Untangling the Web-the impact of Internet use on health care and the physician-patient relationship. Patient Educ Couns 2007;68:218-24.

19. Jeon $\mathrm{YH}$, Flaherty I, Urban $\mathrm{H}$, et al. Qualitative evaluation of evidence-based online decision aid and resources for osteoarthritis management: Understanding patient perspectives. Arthritis Care Res 2019;71:46-55.

20. Gerber BS, Eiser AR. The patient physician relationship in the Internet age: future prospects and the research agenda. $J$ Med Internet Res 2001;3:e15.

21. Murray E, Lo B, Pollack L, et al. The impact of health information on the Internet on health care and the physician-patient relationship: national U.S. survey among 1.050 U.S. physicians. J Med Internet Res 2003;5:e17.

22. Butler L, Foster NE. Back pain online: a cross-sectional survey of the quality of web-based information on low back pain. Spine 2003;28:395-401.

23. Hendrick PA, Ahmed OH, Bankier SS, et al. Acute low back pain information online: an evaluation of quality, content accuracy and readability of related websites. Man Ther 2012;17:318-24.

24. Li L, Irvin E, Guzmán J, et al. Surfing for back pain patients: the nature and quality of back pain information on the Internet. Spine 2001;26:545-57.
25. Nielsen M, Jull G, Hodges PW. Information needs of people with low back pain for an online resource: a qualitative study of consumer views. Disabil Rehabil 2014;36:1085-91.

26. Chan AW, Tetzlaff JM, Altman DG, et al. SPIRIT 2013 statement: defining standard protocol items for clinical trials. Ann Intern Med 2013;158:200-7.

27. Williams CM, Maher CG, Latimer J, et al. Efficacy of paracetamol for acute low-back pain: a double-blind, randomised controlled trial. Lancet 2014;384:1586-96.

28. Osborne RH, Batterham RW, Elsworth GR, et al. The grounded psychometric development and initial validation of the Health Literacy Questionnaire (HLQ). BMC Public Health 2013;13:658.

29. Roland M, Morris R. A study of the natural history of back pain. Part I: development of a reliable and sensitive measure of disability in lowback pain. Spine 1983;8:141-4.

30. Richardson J, lezzi A, Khan M, et al. Data used in the development of the AQoL-8D (PsyQoL) Quality of Life Instrument: In. Centre for Health Economics MU. Melbourne: Centre for Health Economics, Monash University, 2009.

31. Integrated addendum to ICH E6(R1): Guideline for good clinical practice E6(R2), 2016.

32. Nielsen M, Jull G, Hodges PW. Designing an online resource for people with low back pain: health-care provider perspectives. Aust $J$ Prim Health 2016;22:159-66. 